\title{
Transport on flexible Rydberg aggregates using circular states
}

\author{
M. M. Aliyu, ${ }^{1}$ A. Ulugöl, ${ }^{1}$ G. Abumwis, ${ }^{1,2}$ and S. Wüster ${ }^{1,3, *}$ \\ ${ }^{1}$ Department of Physics, Bilkent University, Ankara 06800, Turkey \\ ${ }^{2}$ Max Planck Institute for the Physics of Complex Systems, Nöthnitzer Strasse 38, 01187 Dresden, Germany \\ ${ }^{3}$ Department of Physics, Indian Institute of Science Education and Research, Bhopal, Madhya Pradesh 462 023, India
}

(Received 18 July 2018; published 1 October 2018)

\begin{abstract}
Assemblies of interacting Rydberg atoms show promise for the quantum simulation of transport phenomena, quantum chemistry, and condensed-matter systems. Such schemes are typically limited by the finite lifetime of Rydberg states. Circular Rydberg states have the longest lifetimes among Rydberg states but lack the energetic isolation in the spectrum characteristic of low-angular-momentum states. The latter is required to obtain simple transport models with few electronic states per atom. Simple models can, however, even be realized with circular states by exploiting dipole-dipole selection rules or external fields. We show here that this approach can be particularly fruitful for scenarios where quantum transport is coupled to atomic motion, such as adiabatic excitation transport or quantum simulations of electron-phonon coupling in light harvesting. Additionally, we explore practical limitations of flexible Rydberg aggregates with circular states and to which extent interactions among circular Rydberg atoms can be described using classical models.
\end{abstract}

DOI: 10.1103/PhysRevA.98.043602

\section{INTRODUCTION}

We refer to flexible Rydberg aggregates as assemblies of Rydberg atoms that exhibit excitation transport or collective exciton states and are mobile in a possibly restricted geometry [1]. They exhibit links between motion, excitation transport and coherence [2-5], and spatially inflated BornOppenheimer surfaces for the simulation of characteristic phenomena from the nuclear dynamics of complex molecules [6-10].

Most related experiments [11-16] and theory in this direction have so far focused on aggregates based on Rydberg states with low angular momenta, $l=0,1,2$, due to the possibility of direct excitation and the energetic isolation provided by the energy gap to the nearest other states. For example, $\mid E(|n=49, d\rangle)-E(|n=50, p\rangle) \mid=18.9 \mathrm{GHz}$ in ${ }^{87} \mathrm{Rb}$, which can be much larger than energy scales accessible by Rydberg aggregate dynamics. Here $n$ is the principal quantum number. However, inertia and spontaneous decay limit realistic flexible Rydberg aggregate sizes to less than $\sim$ 4-10 atoms for these low-angular-momentum states.

Rydberg atomic properties are qualitatively changed in circular states, where angular momentum is maximized to $l=n-1$ and pointing along the quantization axis $m=l$, or nearby $l=n-2, m=l=n-2$. Most notably, circular states can have orders of magnitude larger lifetimes than low- $l$ states, ranging into seconds. This has, for example, been essential in their use for quantum-state tomography in cavity quantum electrodynamics [17-21] and has recently attracted attention in the context of quantum computing [22,23] or quantum simulations of spin systems [24]. The price paid for the larger lifetime is a substantially more involved excitation

\footnotetext{
*sebastian@iiserb.ac.in
}

process, which has nonetheless been demonstrated also in an ultracold context [25-28].

Here we determine the utility of a regular assembly of atoms in circular Rydberg states for studies of excitation- and angular-momentum transport as well as a platform for flexible Rydberg aggregates. When working in the quasihydrogenic manifold of circular states, the many-body electronic Hilbert space can no longer be conveniently simplified based on energetic separation of undesired states. However, dipoledipole selection rules can still allow simple aggregate state spaces consisting of only the two nearest to circular states listed above, where we will study two choices. These both differ from the electronic states considered in [24] (in the $n, n+2$ manifolds), in that interactions are direct and no two-photon transition is required. We then focus strongly on the implications for exploiting atomic motion.

We theoretically demonstrate clean back-and-forth transfer of angular momentum within a Rydberg dimer due to the underlying Rabi oscillations between circular states. We also show that in this regime transport can be described both quantum-mechanically and classically, showing good agreement. Interactions between Rydberg atoms in circular states thus might be a further interesting avenue for studies of the quantum-classical correspondence principle with Rydberg atoms [29-34]. Misalignment of the Rydberg aggregate and the electron orbits is shown to cause decreased contrast of the angular-momentum oscillations, which can, however, be suppressed with small electric fields, as also discussed in [24] for a different choice of states.

We finally explore accessible parameter spaces for Rydberg aggregates based on circular states with the primary focus on flexible Rydberg aggregates (atomic motion), taking into account the main limitations, primarily finite lifetime, and adjacent $n$-manifold mixing for too close atomic proximity. We find that flexible aggregates based on circular states offer 
significantly favorable combinations of lifetime and duration of motional dynamics, despite the weaker interactions, compared to aggregates based on low-lying angular-momentum states. The number of aggregate atoms could thus be increased to about $N_{\text {agg }}=50$.

This article is organized as follows: In Sec. II we introduce circular state atoms and their interactions, leading to a model of excitation transfer on a flexible Rydberg chain. Angular-momentum Rabi oscillations in a circular Rydberg dimer are presented in Sec. III and compared to their classical counterpart. The parameter regimes appropriate for the model in Sec. II C are investigated in Sec. IV and then demonstrated in Sec. V with an example for angular-momentum transport in a large flexible aggregates.

\section{RYDBERG ATOMS IN CIRCULAR STATES}

Consider an electronic Rydberg state with principal quantum number $n \gg 10$ of an alkali atom, e.g., ${ }^{87} \mathrm{Rb}$. For a given $n$, we concentrate on the circular or almost circular states with the two highest allowed values of angular momentum $l=(n-1),(n-2)$. In both cases, angular momentum shall point as much as possible along the quantization axis, with azimuthal quantum number $m=+l$. In the following, we write triplets of quantum numbers $|n, l, m\rangle$ for electronic states of atoms. Then our states of main interest are $|a\rangle=$ $|n,(n-1),(n-1)\rangle$ and $|b\rangle=|n,(n-2),(n-2)\rangle$, the circular and next-to-circular states in the principal-quantumnumber manifold $n$. They can be interpreted in terms of Bohr-like orbits, with the electron encircling the nucleus on a circular (or very slightly elliptical) orbit, giving rise to the electron probability densities shown in Fig. 1(b), via their isosurfaces, for the quantization axis along $\hat{z}$.

We will additionally consider a further third state $|c\rangle=$ $|n+1, n, n\rangle$, the fully circular one in the next-higher $n$ manifold; all states are sketched in Fig. 1(a).

\section{A. Effective lifetimes}

The change of angular momentum $\Delta l=l_{2}-l_{1}$ in a spontaneous electric dipole transition from state 1 to state 2 must fulfill $|\Delta l|=1$; hence circular states must decay towards the ground state through radiative cascades via the nearestangular-momentum state and thus exhibit much longer radiative lifetimes $\tau$ in vacuum than lower-angular-momentum (Rydberg) states. At $T=0$ we can use the formula [22,35]

$$
\tau_{0}=\frac{24 \pi \epsilon_{0} \hbar^{4} c^{3}}{\left[E_{H}^{3} a_{0}^{2} e^{2}\right]} \frac{(2 n-1)^{4 n-1}}{2^{4 n+1} n^{2 n-4}(n-1)^{2 n-2}}
$$

for the vacuum lifetime of a circular state in the manifold $n$, which is based on the rate for the first transition of this cascade. In (1) $E_{H}$ is the Hartree energy and $a_{0}$ the Bohr radius. However, $\tau_{0}$ then gets shortened to an effective lifetime $\tau$ by black-body radiation (BBR) at temperature $T$, which accelerates the first step of the cascade by stimulated transitions and may even redistribute electronic population to higher energy states when BBR absorption occurs. We can estimate

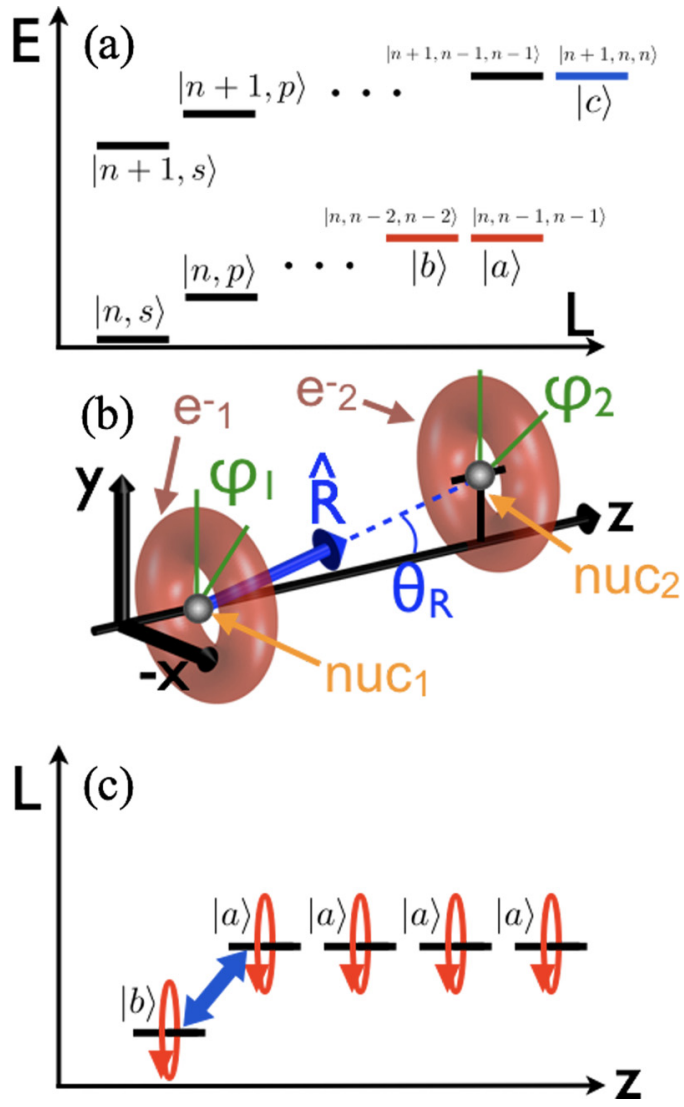

FIG. 1. (a) Schematic diagram of energy $E$ vs angular momentum $L$ for low-angular-momentum vs high-angular-momentum Rydberg states. We highlight the special states relevant for this article $|a\rangle,|b\rangle,|c\rangle$, defined in the text. (b) Schematic shape of the electron probability distribution (tagged with $e_{1,2}^{-}$) for two atoms in circular states with angular momentum pointing fully along the quantization axis $\hat{z}$. Electron orbits are reminiscent of a circular planetary orbit (red toroidal shape). We also indicate nuclear positions and the unit vector along the interatomic axis $\hat{\mathbf{R}}$, its angle with the quantization axis $\theta_{R}$, and orbital angles for classical electron positions $\varphi_{1,2}$. (c) Controlled-angular-momentum transport on a chain of Rydberg atoms along the $z$ axis can proceed using only two single-atom states, $|a\rangle,|b\rangle$, among the high-angular-momentum manifold.

$\tau$, for $T$ in degrees Kelvin, by

$$
\tau=(14.7 \mu \mathrm{s}) \frac{n^{2}}{T},
$$

derived in [36] by using sum rules. For the state $|53,52,52\rangle$, considered later in Fig. 2 of this article, formula Eq. (1) yields a lifetime of $\tau=38 \mathrm{~ms}$ at $T=0$ but Eq. (2) an effective lifetime $\tau=138 \mu$ s at $T=300 \mathrm{~K}$.

\section{B. Binary interactions}

While long lifetimes are an attractive feature for quantum simulations involving Rydberg atoms, such simulations typically rely also on a small accessible electronic state space per atom, such that each atom can, for example, be considered as a (pseudo) spin-1/2 or spin-1 system. This can be realized by Rydberg $|s\rangle(l=0)$ or $|p\rangle(l=1)$ states of the same principal quantum number, provided the energy gap to the $|d\rangle$ 

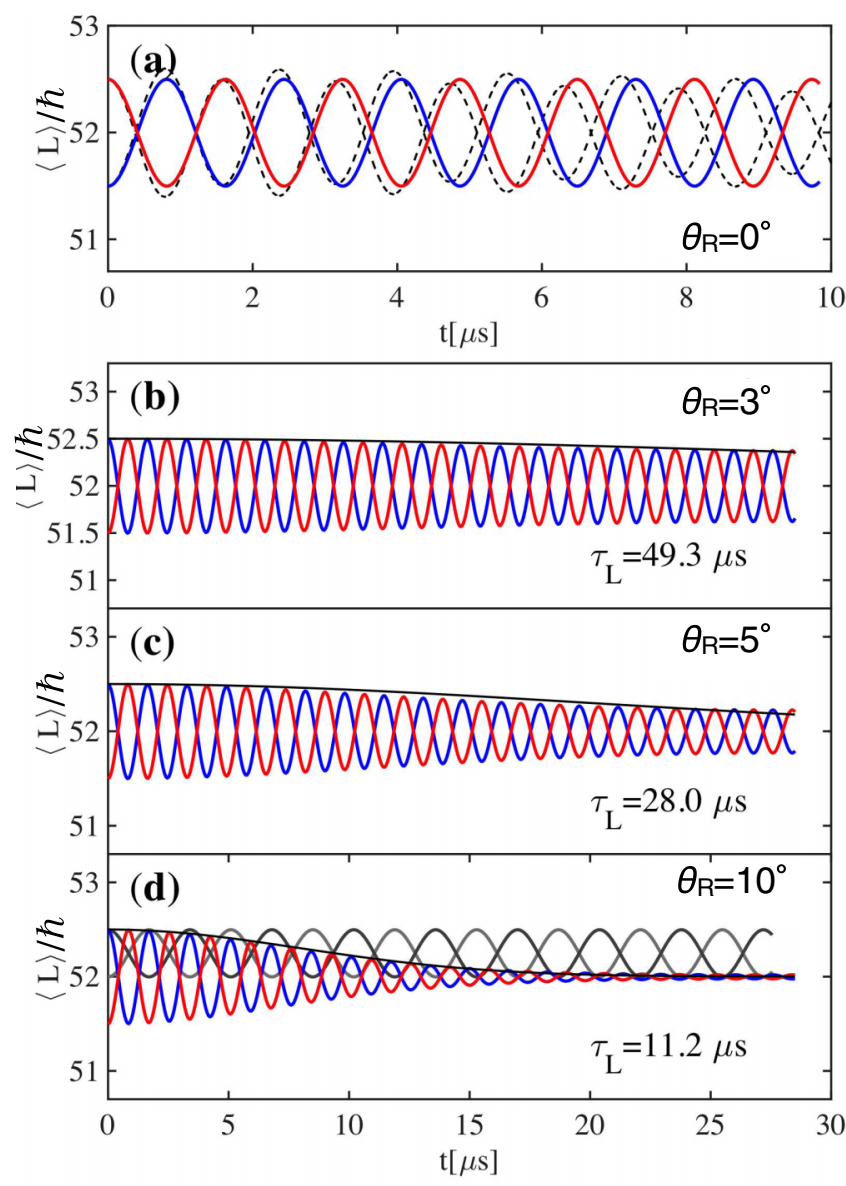

FIG. 2. Angular-momentum transport in a dimer of $n=53$ circular state Rydberg atoms, separated by $R=10 \mu \mathrm{m}$, after initialization in $|\Psi(0)\rangle=|a b\rangle$. Solid lines show the quantum-mechanical results for the angular momentum one for each atom $\left\langle\hat{L}_{1}\right\rangle$ (red, starting at $\left\langle\hat{L}_{1}\right\rangle=52.5$ ) and $\left\langle\hat{L}_{2}\right\rangle$ (blue, starting at $\left\langle\hat{L}_{2}\right\rangle=51.5$ ). Black dashed lines are the corresponding angular momenta from the classical Newton's equations, see Appendix C. In (a), electron orbital planes for state $|a\rangle$ are perfectly normal to the interatomic axis. The angle $\theta_{R}$ between $\hat{R}$ and $\mathbf{z}$ [see Fig. 1(b)] is $\theta_{R}=0^{\circ}$. (b) Quantum-mechanical angular momenta for a misaligned dimer with $\theta_{R}=3^{\circ}$. The solid line is a fit on the envelope as discussed in the text. (c) $\theta_{R}=5^{\circ}$. (d) $\theta_{R}=10^{\circ}$. The gray lines without reduction of oscillation amplitude show the corresponding result in the presence of a small electric field, see text.

state is larger than the dynamical energy scales of the problem, which is frequently the case. In contrast, the high-angularmomentum states become essentially degenerate approaching hydrogen states, so simple energetic inaccessibility can no longer be exploited.

However, in principle, interactions can be designed such that still only two circular Rydberg states per atom play a role. This becomes clear by inspection of the dipole-dipole coupling matrix elements, see Appendix A and Refs. [37-39], for example. These couple only two-body states with the same total azimuthal quantum number $M=m_{1}+m_{2}$, as long as the quantization axis $\hat{\mathbf{z}}$ is oriented along the interatomic separation $\mathbf{R}=\mathbf{x}_{2}-\mathbf{x}_{1}$, where $\mathbf{x}_{1,2}$ are the coordinates of the nuclei in the two interacting atoms. In that case we have $\hat{\mathbf{z}}=$
$\hat{\mathbf{R}}$, where $\hat{\mathbf{R}}=\mathbf{R} /|\mathbf{R}|$. Dipole-dipole interactions (A1) then couple the two pair states $|a b\rangle,|b a\rangle$. However, since these are the only pair states with $M=2 n-3$ for the principalquantum-number $n$ manifold, they form a closed subspace, as long as interactions are weak enough not to cause mixing of adjacent $n$ manifolds.

It is the main objective of this article to explore the limitations of this simple picture. To this end, we consider the more complete Rydberg-Rydberg interactions that arise when taking into account more states and imperfect axis alignment or adjacent $n$-manifold mixing. For this we generate a Rydberg dimer Hamiltonian $\hat{H}_{\text {pair }}$ in matrix form for a fixed atomic separation $\mathbf{R}$ and a large range of pair states $\left|(n, l, m)_{1}\left(n^{\prime}, l^{\prime}, m^{\prime}\right)_{2}\right\rangle$ in the energetic vicinity of those of interest. In the state notation, $(n, l, m)_{k}$ are quantum numbers pertaining to atom $k$. Ingredients of the Hamiltonian are all noninteracting pair energies and matrix elements of the dipole-dipole interactions, as discussed in Appendix A.

We firstly extract dipole-dipole interactions such as $\left\langle b a\left|\hat{H}_{\text {pair }}\right| a b\right\rangle \equiv C_{3}^{(a b)} / R^{3}$, with $R=|\mathbf{R}|$, see also Appendix $\mathrm{B}$. Second, we determine van der Waals interactions in state $|a a\rangle$ by the diagonalization

$$
\hat{H}_{\text {pair }}(\mathbf{R})\left|\phi_{n}(\mathbf{R})\right\rangle=V_{n}(\mathbf{R})\left|\phi_{n}(\mathbf{R})\right\rangle .
$$

The interaction potential $V_{n}(\mathbf{R})$ for which $\left|\phi_{n}(\mathbf{R})\right\rangle \rightarrow|a a\rangle$ for $R \rightarrow \infty$ is then fitted with $V_{n}(\mathbf{R}) \approx C_{6}^{(a a)} / R^{6}+V_{n 0}$ to infer $C_{6}^{(a a)}$.

For simplicity, we neglect spin-orbit interactions throughout this article. Their presence will not cause large quantitative or qualitative changes from the conclusions reached here.

\section{Many-body interactions in flexible Rydberg aggregates}

Armed with binary interactions inferred as discussed above, we can now reduce the effective electronic state space per atom to include only two states. This then enables us to easily treat a larger number of atoms.

We consider a multiatom chain as sketched in Figs. 1(b) and 1(c), where all atoms are as much as possible aligned with the quantization axis $\hat{\mathbf{z}}$. While the angle $\theta_{R}$ between the quantization axis and internuclear axis $\hat{\mathbf{R}}$ is ideally $\theta_{R}=0$, we will later consider alignment imperfections $\theta_{R} \neq 0$. In the ideal case, a single "excitation" in the state $|b\rangle$ can migrate through coherent quantum hops on a chain of circular Rydberg atoms in $|a\rangle$, as sketched in Fig. 1(c).

Note that creating an initial state such as shown, involving two different circular states, poses additional challenges not covered by protocols experimentally demonstrated so far. These only manipulate all atoms in an identical fashion. Possible solutions allowing atom-specific manipulation may have to utilize electric field gradients and sequential optical excitation for atom-selective addressing and could employ optimal coherent control [40].

A setup as in Fig. 1(c) realizes a Rydberg aggregate [1]. Since the number of excitations is conserved, we can describe the aggregate in the basis $\left|\pi_{n}\right\rangle=|a a \cdots b \cdots a a\rangle$, where only the $n$th atom is in the next-to-circular state $|b\rangle$ and all others are in $|a\rangle$. This is called the single-excitation manifold. 
The effective electronic Hamiltonian can then be written as

$$
\begin{gathered}
\hat{H}_{\mathrm{eff}}(\mathbf{X})=\sum_{n \neq m}^{N} \frac{C_{3}^{(a b)}}{X_{n m}^{3}}\left|\pi_{n}\right\rangle\left\langle\pi_{m}\right|+E(\mathbf{X}) \mathbb{1}, \\
E(\mathbf{X})=\frac{1}{2} \sum_{j \neq \ell} \frac{C_{6}^{(a a)}}{X_{j \ell}^{6}},
\end{gathered}
$$

where the vector $\mathbf{X}=\left[\mathbf{x}_{1}, \mathbf{x}_{2}, \mathbf{x}_{3} \ldots\right]$ groups all the individual positions $\mathbf{x}_{n}$ of our $N$ atoms, and $X_{n m}=\left|\mathbf{x}_{n}-\mathbf{x}_{m}\right|, \mathbb{1}$ is the electronic identity matrix $\mathbb{1}=\sum_{n}\left|\pi_{n}\right\rangle\left\langle\pi_{n}\right|$. The first term in (4) allows excitation transport as discussed above and the second represents van der Waals (vdW) interactions between atoms in the $|a\rangle$ state. For simplicity we assumed $C_{6}^{(a b)} \approx$ $C_{6}^{(a a)}$. Typically the dipole-dipole interactions dominate vdW interactions in parameter regions where $C_{6}^{(a b)} \neq C_{6}^{(a a)}$ would make a difference; however, see [10] for counterexamples.

To describe a flexible aggregate with mobile atoms we solve

$$
\hat{H}_{\text {eff }}(\mathbf{X})\left|\varphi_{n}(\mathbf{X})\right\rangle=U_{n}(\mathbf{X})\left|\varphi_{n}(\mathbf{X})\right\rangle
$$

and obtain the excitonic Born-Oppenheimer surfaces $U_{n}(\mathbf{X})$ that govern the atomic motion, see [1].

\section{RYDBERG DIMER WITH CIRCULAR STATES}

We begin to study angular-momentum transport between a pair of Rydberg atoms in circular states for a simple dimer shown in Fig. 1(b). This allows us to still use the Hamiltonian $\hat{H}_{\text {pair }}$ based on a larger number of electronic states per atom. We employ the time-dependent Schrödinger equation (TDSE) $i \hbar \frac{\partial}{\partial t}|\Psi\rangle=\hat{H}_{\text {pair }}|\Psi\rangle$, where the Hamiltonian is constructed as discussed in Sec. II and Appendix A. Within that space

$$
|\Psi(t)\rangle=\sum_{n l m, n^{\prime} l^{\prime} m^{\prime}} c_{n l m, n^{\prime} l^{\prime} m^{\prime}}(t)\left|(n l m)_{1}\left(n^{\prime} l^{\prime} m^{\prime}\right)_{2}\right\rangle,
$$

where $(n l m)_{1}$ are quantum numbers of atom 1 .

The dimer is initialized in the pair state $|\Psi(0)\rangle=$ $|a b\rangle$ for the $n=53$ manifold. As discussed in Sec. II, dipole-dipole interactions cause transitions to the pair state $|b a\rangle$, giving rise to Rabi oscillations in an effective twolevel system, shown in Fig. 2(a). For now, the interatomic axis is perfectly aligned with the quantization axis $\left(\theta_{R}=0\right)$. Physically this implies that Rydberg electron orbitals are orthogonal to the interatomic axis. The figure shows the modulus of electronic angular momentum per atom $\left\langle\hat{L}_{1}\right\rangle=\sum_{n l m, n^{\prime} l^{\prime} m^{\prime}} \hbar \sqrt{l(l+1)}\left|c_{n l m, n^{\prime} l^{\prime} m^{\prime}}\right|^{2},\left\langle\hat{L}_{2}\right\rangle=$ $\sum_{n l m, n^{\prime} l^{\prime} m^{\prime}} \hbar \sqrt{l^{\prime}\left(l^{\prime}+1\right)}\left|c_{n l m, n^{\prime} l^{\prime} m^{\prime}}\right|^{2}$.

\section{A. Quantum-classical correspondence}

The angular-momentum exchange can also be modeled classically, using Newton's equation for the Rydberg electrons, with results shown in black in Fig. 2(a). Further details of these simulations can be found in Appendix C. Already the simple model employed reproduces the quantum results almost quantitatively. This is expected for circular Rydberg states, since the number of de Broglie wavelengths $\lambda_{\mathrm{dB}}$ fitting into one orbital radius $r_{\text {orb }}$ equals $r_{\text {orb }} / \lambda_{\mathrm{dB}}=n$ in BohrSommerfeld theory, which reduces the importance of quantum effects (wave features) for large $n$, in accordance with the correspondence principle.

The result indicates the utility of interactions among circular Rydberg atoms to illustrate the correspondence principle in action. Once verified in more detail, classical simulations could then supplement quantum ones in the regime where each atom accesses a large number of electronic states, which are challenging quantum mechanically.

\section{B. Misalignment of electron orbits and interatomic separation}

In the remainder of Fig. 2, we explore how a misalignment of the circular orbits from the interatomic axis, $\theta_{R}>$ 0 , affects angular-momentum transport. For that case $M=$ $m_{1}+m_{2}$ is no longer conserved in dipole-dipole interactions (see Appendix A). Hence a large number of different azimuthal states $m \neq\{n-1, n-2\}$ become populated. This brings into play additional dipole-dipole interaction matrix elements that cause angular-momentum transfer between the two atoms. Since these differ in magnitude, the overall angular-momentum oscillations in $L_{1,2}$ lose contrast as seen in Figs. 2(b)-2(d). We fitted the envelope of oscillations with $\exp \left[-t^{2} / \tau_{L}^{2}\right]$ and indicated the resultant $\tau_{L}$ in the figures.

Note that even a relative large misalignment such as $\theta=$ $5^{\circ}$ still allows many visible periods of angular-momentum oscillations. The coupling to an undesired azimuthal state can, however, be entirely suppressed by the addition of an electric field. This removes the degeneracy of different $|m|$ states through the dc Stark effect [37]. For Fig. 2(d) we used an electric field amplitude $\mathcal{E}=0.2 \mathrm{~V} / \mathrm{cm}$ and initialized the dimer in $|\Psi(0)\rangle=\left|(53,52,52)_{1}\right\rangle \otimes$ $\left(\left|(53,51,51)_{2}\right\rangle+\left|(53,52,51)_{2}\right\rangle\right) / \sqrt{2} \equiv|a \tilde{b}\rangle$. Note that $|\tilde{b}\rangle=(|53,51,51\rangle+|53,52,51\rangle) / \sqrt{2}$ is the Stark coupled eigenstate corresponding to $|b\rangle$ in the presence of the field. While the Rabi frequency is now reduced by a factor of 2 , since the dipole-dipole interaction couples only the first component of $|\tilde{b}\rangle$ to $|a\rangle$, we regain an effective two-level system. Calculations with electric field were streamlined by solving the TDSE only in the most relevant state space [41]. Suppressing coupling to undesired $m$ states through an external field was explored in detail in [24] for coupled states from different (next-to-adjacent) $n$ manifolds. Here we now extended these concepts to almost circular states from the same $n$ manifold.

\section{Adjacent $\boldsymbol{n}$-manifold mixing}

So far, we explored one limitation of the simple picture in which only circular states $|a b\rangle$ and $|b a\rangle$ are considered, namely, undesired $m$ levels mixing in through atomic misalignment. We have shown that this effect can be suppressed using external electric fields.

Another limitation of the simple model arises at too short distances, where state manifolds that differ in principal quantum number $n$ are shifted into each other through strong interactions. We show the resultant spectrum in Fig. 3, for a much lower principal quantum number $(n=20)$ than used in Fig. 2, due to computational reasons.

For demonstration, the figure also shows the detrimental effect on angular-momentum transport through this state 


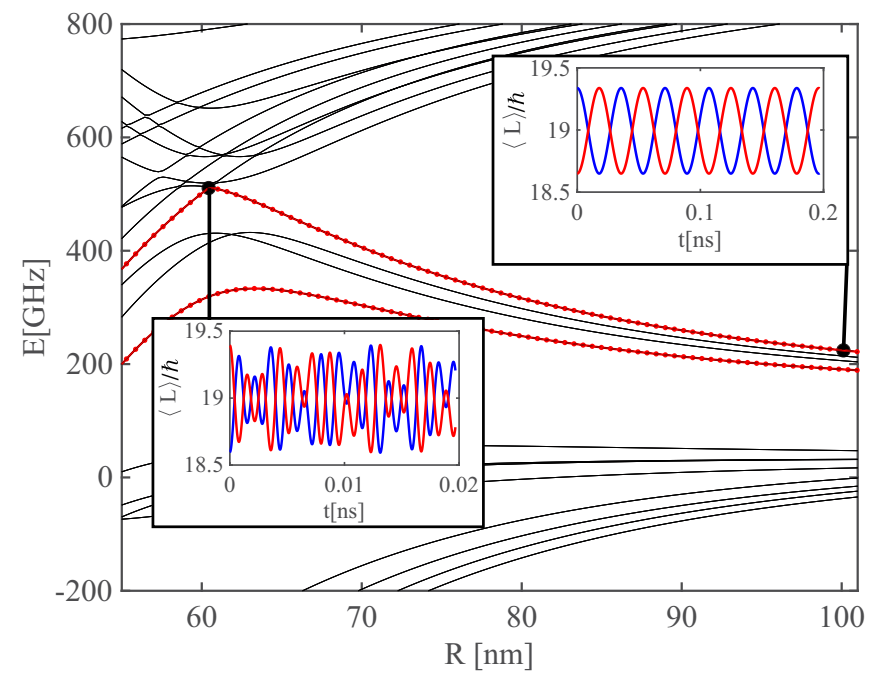

FIG. 3. Interaction potentials $V_{n}(R)$ of a circular Rydberg dimer near $n=20$ at close proximity, see Eq. (3). The reduced Hilbert space contained all states with $n=19,20,21$ and $l=18,19,20$. The simple effective-state picture involving only two circular states $|a\rangle$ and $|b\rangle$ that couple via dipole-dipole interactions to $(|a b\rangle \pm$ $|b a\rangle) / \sqrt{2}$ (red lines with dots) breaks down once neighboring $n$ manifolds begin to merge into each other at around $R=60 \mathrm{~nm}$. The insets show angular-momentum transport from initial states as in Fig. 2(a) at the indicated separations.

mixing. The right inset shows angular-momentum oscillations that are regular at distances where adjacent $n$ manifolds are energetically separate. However, even here the initial state is composed of eigenstates from (3) according to $\left(\left|\phi_{a b}(\mathbf{R})\right\rangle+\right.$ $\left.\left|\phi_{b a}(\mathbf{R})\right\rangle\right) / \sqrt{2}$, where $\left|\phi_{a b}\right\rangle$ denotes the eigenstate of $\hat{H}_{\text {pair }}$ that has the largest overlap with $|a b\rangle$. Oscillations finally become irregular at separations where adjacent $n$ manifolds mix, shown in the left inset, even when constructing an initial state from four eigenstates similar to the construction above. This effect imposes a minimal separation $d_{\min }$ for atoms in a circular Rydberg aggregate, which we define as the distance at which the dipole-dipole shift exceeds the energetic $n$ manifold separation. The resultant formula is given in Appendix D.

\section{PARAMETER REGIMES FOR CIRCULAR RYDBERG AGGREGATES}

After exploring the limitations of the simple model introduced in Sec. IIC, which are not problematic for the right choice of atomic positions $\mathbf{x}_{n}$, we now proceed to determine interaction parameters required for the model (4) as discussed in Sec. II B.

\section{A. Determination of interaction constants}

For dipole-dipole interactions we extract the matrix elements $\left\langle a b\left|\hat{H}_{\text {pair }}\right| b a\right\rangle$ and $\left\langle a c\left|\hat{H}_{\text {pair }}\right| c a\right\rangle$ from the numerical Hamiltonian and verify the former analytically in Appendix B. Next we consider vdW interactions for two atoms in the state $|a\rangle$ (i.e., the energy of $|a a\rangle$ ). We find these by diagonalizing a suitable Hamiltonian as a function of atomic separation $R$, as
TABLE I. Reference values in interaction parameters for dipoledipole and van der Waals interactions of ${ }^{87} \mathrm{Rb}$ atoms in circular or next-to-circular Rydberg states. Using these parameters, interaction strengths can be found with Eqs. (8)-(10). States $|a\rangle,|b\rangle,|c\rangle$ are sketched in Fig. 1 and defined in Sec. II.

\begin{tabular}{lcc}
\hline \hline & $\tilde{C}_{3}^{(0)}\left[\mathrm{kHz} \mu \mathrm{m}^{3}\right]$ & $\tilde{C}_{6}^{(0)}\left[\mathrm{Hz} \mu \mathrm{m}^{6}\right]$ \\
\hline$|a a\rangle$ & & $2.11 \times 10^{-11}$ \\
$|a b\rangle$ & 2.0 & \\
$|a c\rangle$ & 0.47 & \\
\hline \hline
\end{tabular}

discussed in Sec. II B and Appendix A. All these calculations assume an internuclear axis aligned with the quantization axis $\hat{\mathbf{R}} \| \hat{\mathbf{z}}$, which is enough to determine the scale of interactions in a setting such as Fig. 1(c).

All interactions exhibit a characteristic scaling with principal quantum number $n$ :

$$
\begin{gathered}
C_{6}^{(a a)}=\tilde{C}_{6}^{(0)} n^{12}, \\
C_{3}^{(a b)}=\tilde{C}_{3, a b}^{(0)} n^{3} \quad \text { for }|a b\rangle, \\
C_{3}^{(a c)}=\tilde{C}_{3, a c}^{(0)} n^{4} \quad \text { for }|a c\rangle,
\end{gathered}
$$

which allows their approximate representation in terms of the reference values $\tilde{C}_{k}^{(0)}$ given in Table I. The table distinguishes between dipole-dipole interactions within the same or among adjacent $n$ manifolds. Note that the scaling of interactions with $n$ is different from that encountered for low-lying angular-momentum states, where dipole-dipole interactions scale as $n^{4}$ and van der Waals interactions as $n^{11}$ [37]. vdW interaction strengths from Eq. (8) and Table I for circular states with $n=48$ and $n=50$ are in rough agreement with the values given in [24], the latter calculated at nonzero electric and magnetic fields.

\section{B. Domains for flexible Rydberg aggregates}

Dipole-dipole interactions in the pair $|a c\rangle$ substantially exceed those in $|a b\rangle$ for the relevant high principal quantum numbers $(n>20)$ due to the steeper scaling in $n$. We thus now assume aggregates based on states $\left|\tilde{\pi}_{n}\right\rangle=|a a \cdots c \cdots a a\rangle$, where only the $n$th atom is in the state $|c\rangle$ and all others in $|a\rangle$, replacing the states $\left|\pi_{n}\right\rangle$ in Sec. II C.

With interactions determined, we can follow the approach taken in [1] to delineate parameter regimes in which circular flexible or static Rydberg aggregates are viable, based on a variety of requirements that are listed in detail in Appendix D. The results are shown in Fig. 4. It is clear that the use of circular Rydberg atoms for studies involving atomic motion offers substantial advantages. However, this is the case only in a cryogenic environment at $T \approx 4 \mathrm{~K}$, since black-body redistribution has too detrimental an effect on the lifetime advantage otherwise. Ideas to suppress spontaneous decay by tuning the electromagnetic mode structure with a capacitor could improve this situation further $[24,42,43]$. 

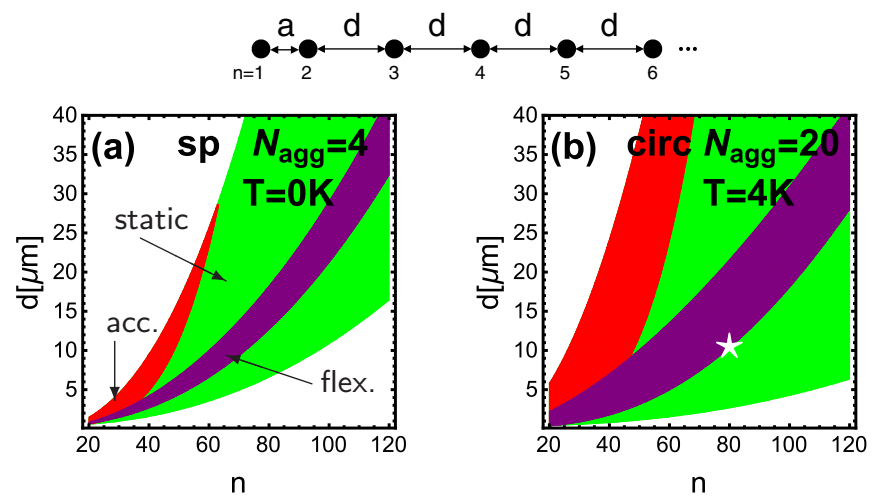

FIG. 4. Parameter domains of static (green and red) vs flexible (violet) Rydberg aggregates for different principal quantum numbers $n$ and nearest-neighbor separations $d$. For the latter we assume geometry as shown on the top, with main nearest-neighbor separation $d$ and shorter initial dislocation $a$. We compare the use of $s p$ Rydberg states in (a) vs circular Rydberg states in (b), where the latter are assumed to be in a cryogenic environment at $T=4 \mathrm{~K}$. Note the substantially different aggregate sizes $N_{\text {agg }}$ assumed for either as indicated. The red shade (marked acc.) indicates where static aggregates exist, however, with atoms that would visibly accelerate during excitation transport. White areas are excluded, either by too short aggregate lifetimes (top) or too close proximities to avoid Rydberg state mixing as in Fig. 3 (bottom). See the text and Appendix D for the precise criteria used. The symbol $(\star)$ in (b) indicates parameters used for our numerical demonstration in Fig. 5.

\section{ANGULAR-MOMENTUM TRANSPORT IN LARGE FLEXIBLE RYDBERG AGGREGATES}

To illustrate the potential of circular state Rydberg aggregates for studying the coupling between atomic motion and excitation transport, we show a quantum-classical simulation of adiabatic excitation transport on a large $\left(N_{\text {agg }}=20\right)$ Rydberg aggregate. Adiabatic excitation transport in Rydberg aggregates was thoroughly discussed in $[2,3,8]$. Briefly, a single excited state is initially coherently shared among two atoms at one end of the chain, that are in much closer proximity $a$ than all others, here $a=5 \mu \mathrm{m}$. These are atoms $n=1,2$ in the sketch on top of Fig. 4. This initial state, $\left|\varphi_{\text {rep }}\right\rangle=$ $(\mid$ caaa $\ldots\rangle+\mid$ acaa $\ldots\rangle) / \sqrt{2}$, is the most repulsive eigenstate in Eq. (6).

The initial repulsion of atoms 1 and 2 causes subsequent repulsive collisions with the remainder of the atoms, the dislocation thus propagating through the chain. The single excitation is carried along with the positional dislocation with high fidelity. This can be traced back to an adiabatic following of the initial dipole-dipole eigenstate $\left|\varphi_{\text {rep }}(\mathbf{X}(t))\right\rangle$ [see Eq. (6)].

We model the process using Tully's surface hopping [44-46], described for our specific purposes in [8,47]. It evolves an electronic aggregate quantum state $\left|\Psi_{\text {agg }}(t)\right\rangle=$ $\sum_{n} c_{n}(t)\left|\pi_{n}\right\rangle$, coupled to the classical Newton equations $m_{\mathrm{Rb}} \ddot{\mathbf{X}}(t)=-\nabla_{\mathbf{X}} U_{s}[\mathbf{X}(t)]$ for motion of rubidium atoms with mass $m_{\mathrm{Rb}}$ on the current Born-Oppenheimer surface $U_{s(t)}$ [see Eq. (6)]. Note that creating the initial electronic state $\left|\varphi_{\text {rep }}\right\rangle$ will pose additional experimental challenges.

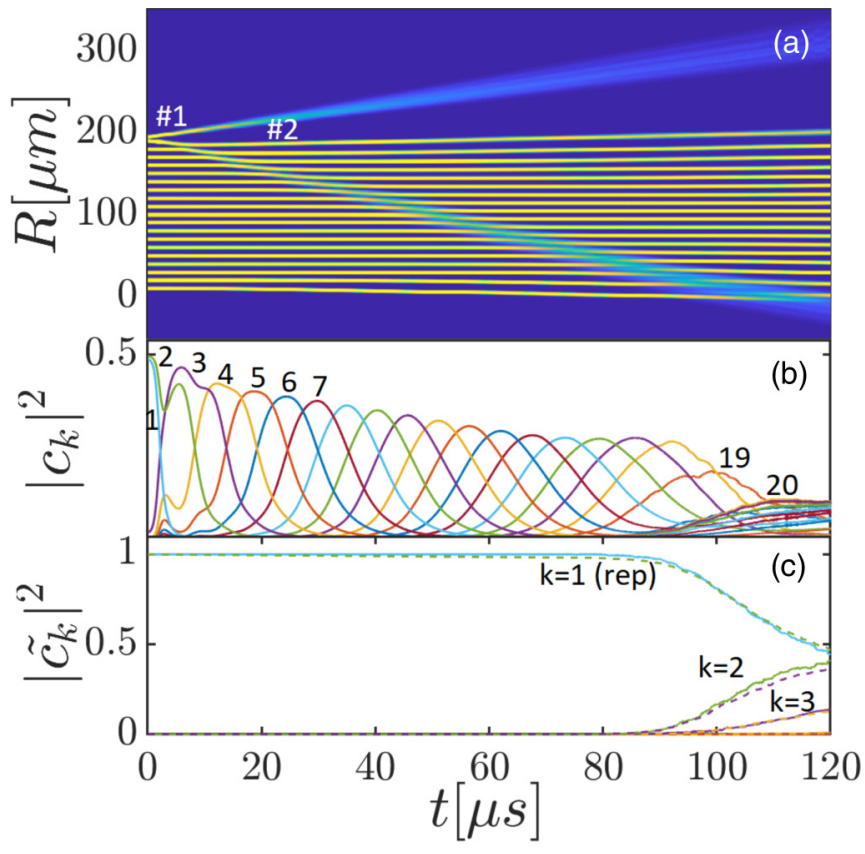

FIG. 5. Adiabatic-angular-momentum transport on a large flexible Rydberg aggregate with $N=20$ atoms arranged in a onedimensional line along $z$ with spacing $d=10 \mu \mathrm{m}$, but the last two atoms only $a=5 \mu \mathrm{m}$ apart. Dynamics proceeds on the repulsive Born-Oppenheimer surface $n=0$. The aggregate is based on circular states $|a\rangle,|c\rangle$ with principal quantum number $n=80$. For that value, the effective lifetime from Eq. (2) for the entire aggregate is $\tau_{\text {agg }}=\tau / N_{\text {agg }} \approx 1.2 \mathrm{~ms}$ at $T=4 \mathrm{~K}$. Each atom has a spatial position uncertainty of $\sigma=0.3 \mu \mathrm{m}$. (a) Total density of atoms, bright (yellow) indicates high density, blue (dark) no density. (b) Excitation amplitudes on each atom $\left|c_{k}\right|^{2}=\left|\left\langle\pi_{k} \mid \Psi(t)\right\rangle\right|^{2}$, with atom number $k$ indicated near each line. We indicate where numbering starts in (a). (c) Populations of system eigenstates $\left|\tilde{c}_{k}\right|^{2}=\left|\left\langle\varphi_{k}(\mathbf{X}) \mid \Psi(t)\right\rangle\right|^{2}$, discussed in Sec. II C, indicating largely adiabatic dynamics.

The parameters used for the simulation are indicated by the white star in Fig. 4, and the (one-dimensional) geometry is sketched on top of that figure. For these parameters, even $N_{\text {agg }}=100$ would still allow end-to-end transport within the lifetime, however, with long simulation times due to the need for matrix diagonalization at each time step.

Proposals in $[2,3,8]$ were limited by spontaneous decay to about eight Rydberg atoms, even when considering the lighter, and thus more easily accelerated, lithium atom. The quantum-classical simulation shown in Fig. 5 highlights that for aggregates made of circular states much larger arrays are possible, even for the heavier but more common rubidium atom, and still show adiabatic excitation transport within the system lifetime, i.e., well before a single black-body redistribution event is expected.

While the multitrajectory average in Fig. 5(b) seems to indicate a loss of fidelity for the excitation transport, this is merely due to the different arrival times for different parts of the many-body wave packet (different trajectories). We inspected many individual quantum-classical trajectories which all show near unit fidelity of excitation transport through the entire chain. 


\section{CONCLUSIONS AND OUTLOOK}

We assess the utility of arrays of Rydberg atoms in circular and nearly circular angular-momentum states for the realization of flexible Rydberg aggregates. While the motion of circular state Rydberg atoms was considered in [24] as a precursory stage during the creation of regular static arrays, in our work freely moving atoms are the primary focus. These will then allow studying the interrelationship between atomic motion and excitation- or angular-momentum transport. Note that the apparatus proposed in [24] would also be highly suitable for such studies.

In a cryogenic environment (suppressing black-body radiation), circular state flexible Rydberg aggregates will allow much larger arrays of atoms to participate in collective motional dynamics, despite their inertia, due to the substantially increased lifetimes. For example, adiabatic excitation transport with high fidelity on chains of as many as $N_{\text {agg }}=50$ atoms appears feasible. In the future we will explore the application of this phenomenon for use as a data bus in circular Rydberg-atom-based quantum computing architectures [22,23].

We also demonstrate a case where interacting circular Rydberg atoms can be quite well described using the classical Newton's equations for the Rydberg electrons in a manifestation of the correspondence principle. Both quantum and classical calculations exhibit comparable coherent angularmomentum oscillations in a pair of circular Rydberg atoms. More detailed comparisons using more involved classicalphase-space distributions and quantum wave packets, larger numbers of atoms, or more involved geometries could be an interesting exploration of the extent of the correspondence principle. A classical treatment of interactions could then benefit from secular perturbation theory techniques also used in planetary orbital mechanics.

\section{ACKNOWLEDGMENTS}

We gratefully acknowledge fruitful discussions with Mehmet Oktel and Michel Brune.

\section{APPENDIX A: CIRCULAR RYDBERG INTERACTIONS}

We assume the interatomic interactions are entirely based on the dipole-dipole component of the electrostatic Hamiltonian (in atomic units)

$$
\hat{H}_{d d}=\frac{\mathbf{r}_{1} \cdot \mathbf{r}_{2}-3\left(\mathbf{r}_{1} \cdot \hat{\mathbf{R}}\right)\left(\mathbf{r}_{2} \cdot \hat{\mathbf{R}}\right)}{R^{3}},
$$

where $\mathbf{r}_{1}$ and $\mathbf{r}_{2}$ denote the position of the Rydberg electron in atoms 1,2 relative to their parent nuclei, and $\hat{\mathbf{R}}=\mathbf{R} / R$ is a unit vector along the interatomic separation $\mathbf{R}=\mathbf{x}_{2}-\mathbf{x}_{1}$, with $R=|\mathbf{R}|$, see Fig. 1(b). We thus ignore wave-function overlap, core polarization, or higher-order multipoles, as is typical for Rydberg-Rydberg interactions.

We then cast (A1) into a matrix form using pair states $\left|n_{1}, l_{1}, m_{1}\right\rangle_{1} \otimes\left|n_{2}, l_{2}, m_{2}\right\rangle_{2}$ in a truncated Hilbert space in which all pair states are energetically close to those for which we want to determine Rydberg-Rydberg interactions. As usual, the position-space representation is written as $\left\langle\mathbf{r}_{1} \mid n_{1}, l_{1}, m_{1}\right\rangle=\mathcal{R}_{n_{1} l_{1}}\left(r_{1}\right) Y_{l_{1}, m_{1}}\left(\theta_{1}, \varphi_{1}\right) / r_{1}$, where $Y$ are spherical harmonics, and $\left(r_{1}, \theta_{1}, \varphi_{1}\right)$ the three-dimensional (3D) spherical polar coordinates of electron 1 with respect to its nucleus.

The matrix elements of (A1) are

$$
\begin{aligned}
& \left\langle n_{1}, l_{1}, m_{1} ; n_{2}, l_{2}, m_{2}\left|\hat{H}_{d d}\right| n_{1}^{\prime}, l_{1}^{\prime}, m_{1}^{\prime} ; n_{2}^{\prime}, l_{2}^{\prime}, m_{2}^{\prime}\right\rangle \\
& =-8 \pi \sqrt{\frac{2 \pi}{15}} \frac{d_{n_{1}, l_{1} ; n_{1}^{\prime}, l_{1}^{\prime}}}{R_{n_{2}, l_{2} ; n_{2}^{\prime}, l_{2}^{\prime}}^{3}} \\
& \quad \times \sum_{m_{a}, m_{b}} \sum_{\mu=-2}^{2} Y_{l=2, \mu}^{*}\left(\theta_{R}, \varphi_{R}\right)\left\langle 1 m_{1}, 1 m_{2} \mid 2 \mu\right\rangle \\
& \quad \times\left\langle l_{1}, m_{1}\left|Y_{1 m_{1}}\right| l_{1}^{\prime}, m_{1}^{\prime}\right\rangle\left\langle l_{2}, m_{2}\left|Y_{1 m_{2}}\right| l_{2}^{\prime}, m_{2}^{\prime}\right\rangle,
\end{aligned}
$$

see also [38]. Here $\theta_{R}, \varphi_{R}$ are the polar angles of $\hat{\mathbf{R}}$ in the 3D spherical coordinate system defining $n, l, m,\left\langle 1 m_{1}, 1 m_{2} \mid 2 \mu\right\rangle$ the Clebsch-Gordan coefficient coupling two constituent angular momenta $\left(l=1, m=m_{1,2}\right)$ to a total angular momentum $(L=2, M=\mu)$, and the integrals in the last line involve now a single electronic coordinate and three spherical harmonics each.

Evaluating these as in [48], we use

$$
\begin{aligned}
\left\langle l_{1}, m_{1}\left|Y_{1 m_{1}}\right| l_{1}^{\prime}, m_{1}^{\prime}\right\rangle & \\
= & (-1)^{m_{1}} \sqrt{\frac{3\left(2 l_{1}+1\right)\left(2 l_{1}^{\prime}+1\right)}{4 \pi}} \\
& \times\left(\begin{array}{ccc}
l_{1} & l_{1}^{\prime} & 1 \\
0 & 0 & 0
\end{array}\right)\left(\begin{array}{ccc}
l_{1}^{\prime} & l_{1} & 1 \\
m_{1}^{\prime} & -m_{1} & m_{1}
\end{array}\right),
\end{aligned}
$$

where terms in brackets denote Wigner $3 j$ symbols.

The $d_{n_{1}, l_{1} ; n_{1}^{\prime}, l_{1}^{\prime}}=\int_{0}^{\infty} r \mathcal{R}_{n_{1}, l_{1}}(r) \mathcal{R}_{n_{1}^{\prime}, l_{1}^{\prime}}(r) d r$ in (A2) are radial matrix elements, determined via the Numerov method including modifications of the Coulomb potential due to the core as in [49]. To avoid instabilities, the solutions $\mathcal{R}(r)$ are set to zero inside the inner classical turning point for large $l$.

When considering interactions within an external electric field of strength $\mathcal{E}$, we describe the field through single-body matrix elements

$$
\begin{aligned}
-\left\langle n, l, m|\mathcal{E} e \hat{\mathbf{z}}| n^{\prime}, l^{\prime}, m^{\prime}\right\rangle \\
=-d_{n, l ; n^{\prime}, l^{\prime}} \mathcal{E} e \sqrt{\frac{3(2 l+1)\left(2 l^{\prime}+1\right)}{4 \pi}} \\
\quad \times\left(\begin{array}{ccc}
l & l^{\prime} & 1 \\
0 & 0 & 0
\end{array}\right)\left(\begin{array}{ccc}
l^{\prime} & l & 1 \\
m^{\prime} & -m & 0
\end{array}\right) .
\end{aligned}
$$

To obtain vdW interaction potentials, the resultant dimer Hamiltonian $\hat{H}_{\text {pair }}=\hat{H}_{0}+\hat{H}_{d d}$ is diagonalized as a function of separation $R$, see Eq. (3) and, e.g., Fig. 3. Here, the noninteracting Hamiltonian is $\hat{H}_{0}=\sum_{\boldsymbol{\alpha}_{1}, \boldsymbol{\alpha}_{2}}\left(E_{\boldsymbol{\alpha}_{1}}+\right.$ $\left.E_{\boldsymbol{\alpha}_{2}}\right)\left|\boldsymbol{\alpha}_{1} \boldsymbol{\alpha}_{2}\right\rangle\left\langle\boldsymbol{\alpha}_{1} \boldsymbol{\alpha}_{2}\right|$, where the $\boldsymbol{\alpha}$ group all electronic labels, such as $\alpha_{1}=\{n, l, m\}$ with $E_{\alpha_{1}}=E_{n_{1}, l_{1}, m_{1}}=-\mathrm{Ry} /\left(n_{1}-\right.$ $\left.\delta_{n_{1}, l_{1}}\right)^{2}$. Then Ry is the Rydberg constant and $\delta_{n, l}$ the quantum defect taken from $[49,50]$. For transport simulations, the restricted basis Hamiltonian is constructed at a fixed separation $R_{0}$ and then used in the time-dependent Schrödinger equation.

A recent numerical package for these sorts of calculations is described in [51]. For low-lying state interaction, also perturbation theory can be used [52]. 


\section{APPENDIX B: CALCULATION OF DIPOLE-DIPOLE INTERACTION CONSTANTS}

For circular states of alkali atoms, the wave-function overlap with the core becomes so small that the use of hydrogen wave functions $\Psi\left(r_{k}, \theta_{k}, \varphi_{k}\right)=\mathcal{R}_{n l}\left(r_{k}\right) Y_{l m}\left(\theta_{k}, \varphi_{k}\right) / r_{k}$, where $k \in\{1,2\}$ numbers the atom, becomes highly justified. We can then determine, e.g., $C_{3}^{(a b)}$ coefficients from Eq. (A1) by inserting the appropriate sets of quantum numbers into the matrix element $\mathcal{M}=\left\langle a b\left|\hat{H}_{\mathrm{dd}}\right| b a\right\rangle$ between hydrogen states.

Since $\hat{\mathbf{R}} \| \hat{\mathbf{z}}$ we have $\theta_{R}=0$. In that case, only $Y_{l=2,0}^{*}\left(\theta_{R}=\right.$ $0, \varphi)$ in the sum over $\mu$ is nonzero, and out of the options for $m_{1}+m_{1}^{\prime}=0$ only one set fulfills the remaining angularmomentum selection rules in (A2), yielding the integral

$$
I=\langle 1,1 ; 1,-1 \mid 2,0\rangle\left\langle a\left|Y_{1,1}\right| b\right\rangle\left\langle b\left|Y_{1,-1}\right| a\right\rangle,
$$

which results in

$$
\begin{aligned}
I= & \frac{(-1)^{2(2 n-3)}(2 n-1)(2 n-3)(2 n-2) !(2 n-4) !}{2^{2(2 n-3)}((n-1) !(n-2) !)^{2}} \\
& \times\left(\frac{1}{4 \pi}\right)^{2}\left(-\frac{3(2 \pi)^{2}}{8 \pi \sqrt{6}}\right)\left(\frac{\sqrt{\pi} \Gamma(n)}{\Gamma\left(n+\frac{1}{2}\right)}\right)^{2},
\end{aligned}
$$

where $\Gamma(n)$ is the Gamma function. Using $Y_{2,0}^{*}\left(\theta_{R}=0, \varphi\right)=$ $\sqrt{\frac{5}{4 \pi}}$ and the radial matrix element

$$
d_{n(n-1) ; n(n-2)}=-\frac{3 n}{2} \sqrt{(2 n-1)},
$$

we finally reach

$$
\begin{aligned}
\mathcal{M}= & -\frac{8 \pi}{R^{3}} \sqrt{\frac{2 \pi}{15}} \sqrt{\frac{5}{4 \pi}}\left(\frac{-3 n}{2} \sqrt{(2 n-1)}\right)^{2} \\
& \times \frac{(-1)^{2(2 n-3)}(2 n-1)(2 n-3)(2 n-2) !(2 n-4) !}{2^{2(2 n-3)}((n-1) !(n-2) !)^{2}} \\
& \times\left(\frac{1}{4 \pi}\right)^{2}\left(-\frac{3(2 \pi)^{2}}{8 \pi \sqrt{6}}\right)\left(\frac{\sqrt{\pi} \Gamma(n)}{\Gamma\left(n+\frac{1}{2}\right)}\right)^{2} .
\end{aligned}
$$

See [22] for analytical results for the $|a a\rangle \leftrightarrow\left|c c^{\prime}\right\rangle$ dipolematrix elements, where $\left|c^{\prime}\right\rangle=|n-1, n-2, n-2\rangle$.

\section{APPENDIX C: CLASSICAL SIMULATIONS OF THE RYDBERG DIMER}

In the classical simulations, we adopted the BohrSommerfeld atomic model to mimic the orbital behavior by using elliptical orbits for a classical point electron. Initial positions and velocities are drawn from a random distribution that respects the target quantum numbers via energy and (angular momentum):

$$
\begin{gathered}
E_{n}=-\frac{e^{4} m_{e}}{32 \pi^{2} \epsilon_{0}^{2} \hbar^{2}} \frac{1}{n^{2}}, \\
L_{m}=\hbar \sqrt{l(l+1)},
\end{gathered}
$$

where $m_{e}$ is the mass of the electron.
In the model, the electron follows an elliptic path and the semimajor $\left(A_{n}\right)$ and semiminor $\left(B_{n l}\right)$ axes are defined as

$$
A_{n}=\frac{4 \pi \epsilon_{0} \hbar^{2}}{m_{e} e^{2}} n^{2}, \quad B_{n l}=\frac{l}{n} A_{n} .
$$

In the simulation, nuclei of the atoms are assumed to be motionless and the equation of motion for the electrons is

$$
\begin{aligned}
\ddot{\mathbf{r}}_{e i}= & -\frac{e^{2}}{4 \pi \epsilon_{0} m_{e}}\left(\frac{\mathbf{r}_{e i}-\mathbf{r}_{n i}}{\left|\mathbf{r}_{e i}-\mathbf{r}_{n i}\right|^{3}}+\frac{\mathbf{r}_{e i}-\mathbf{r}_{n(i+1)}}{\left|\mathbf{r}_{e i}-\mathbf{r}_{n(i+1)}\right|^{3}}\right. \\
& \left.-\frac{\mathbf{r}_{e i}-\mathbf{r}_{e(i+1)}}{\left|\mathbf{r}_{e i}-\mathbf{r}_{e(i+1)}\right|^{3}}\right),
\end{aligned}
$$

where the index $n i$ is the $i$ th nucleus and the index $e i$ is the $i$ th electron. The notation $(i+1)$ pertains here simply to the adjacent atom in a dimer.

The classical simulation is conducted by numerical evaluation of the equation of motion and averaging the results over random initial positions of the electron on the elliptic orbit. For this we vary in particular the relative orbital phase between the electrons, $\varphi_{2}-\varphi_{1}$, see Fig. 1(b).

The black dashed lines in Fig. 2(a) show finally the ensemble-averaged angular momenta $L_{k}=\left|\overline{\mathbf{L}_{k}}\right|$, where $\mathbf{L}_{k}$ is the angular momentum of electron $k$ with respect to nucleus $k$. The model could be made more sophisticated by incorporating also the out-of-plane distribution of the Rydberg electron evident in Fig. 1(b) or nuclear motion.

\section{APPENDIX D: PARAMETER CONSTRAINTS FOR RYDBERG AGGREGATES}

For the parameter space survey in Sec. IV we have utilized the following mathematical criteria to define when a one-dimensional circular Rydberg atom chain can constitute a useful flexible Rydberg aggregate. We are following the approach of [1].

Validity of the essential-state model. We have seen in Fig. 3 that the essential-state models based on $|a\rangle,|b\rangle$ or $|a\rangle$, $|c\rangle$ break down once adjacent $n$ manifolds begin to mix. We have taken the corresponding distance $d_{\min }$ as the one where $C_{3}^{(a c)}(n) / d_{\min }^{3}=1 /\left(2 n^{2}\right)-1 /\left[2(n+1)^{2}\right]$ (atomic units).

Static aggregates. From (9) we can infer a transfer time (Rabi oscillation period) $T_{\text {hop }}=\pi d^{3} / C_{3}$ for an excitation to migrate from a given atom to the neighboring one if the interatomic spacing is $d$. We have calculated the corresponding time for $N_{\text {hops }}=100$ such transfers, given by $T_{\text {trans }}=$ $N_{\text {hops }} T_{\text {hop }}$, imagining migration along an entire aggregate. We finally require $T_{\text {trans }}$ to be short compared to the system lifetime, which is determined for circular states based on Eq. (2).

Perturbing acceleration. The characteristic time for atom acceleration is $T_{\mathrm{acc}}=\sqrt{\frac{d^{5} m_{R b}}{6 C_{3}^{(a c)}}}$ [1], with mass of the atoms $m_{\mathrm{Rb}}$ and their initial separation $d$. We then color the parameter space red in Fig. 4, where atoms would inadvertently be set into motion due to $4 T_{\text {acc }}<T_{\text {trans }}$.

Flexible aggregates. For flexible aggregates, we assume an equidistant chain with spacing $d$ but the existence of a dislocation on the first two atoms with spacing of only $d_{\text {ini }}=a=d / 2$ to initiate directed motion, similar to Sec. V. 
Hence, $d_{\text {ini }}>d_{\min }$ must be fulfilled, a tighter constraint than $d>d_{\text {min }}$. We can then assess as in [1] whether an excitation- transporting pulse can traverse the chain within the system lifetime.
[1] S. Wüster and J. M. Rost, J. Phys. B 51, 032001 (2018).

[2] S. Wüster, C. Ates, A. Eisfeld, and J. M. Rost, Phys. Rev. Lett. 105, 053004 (2010).

[3] S. Möbius, S. Wüster, C. Ates, A. Eisfeld, and J. M. Rost, J. Phys. B 44, 184011 (2011).

[4] S. Wüster, Phys. Rev. Lett. 119, 013001 (2017).

[5] D. W. Schönleber, A. Eisfeld, M. Genkin, S. Whitlock, and S. Wüster, Phys. Rev. Lett. 114, 123005 (2015).

[6] S. Wüster, A. Eisfeld, and J. M. Rost, Phys. Rev. Lett. 106, 153002 (2011).

[7] K. Leonhardt, S. Wüster, and J. M. Rost, Phys. Rev. Lett. 113, 223001 (2014).

[8] K. Leonhardt, S. Wüster, and J. M. Rost, Phys. Rev. A 93, 022708 (2016).

[9] K. Leonhardt, S. Wüster, and J. M. Rost, J. Phys. B 50, 054001 (2017).

[10] H. Zoubi, A. Eisfeld, and S. Wüster, Phys. Rev. A 89, 053426 (2014).

[11] D. Barredo, H. Labuhn, S. Ravets, T. Lahaye, A. Browaeys, and C. S. Adams, Phys. Rev. Lett. 114, 113002 (2015).

[12] H. Labuhn, D. Barredo, S. Ravets, S. de Léséleuc, T. Macrì, T. Lahaye, and A. Browaeys, Nature (London) 534, 667 (2016).

[13] S. Bettelli, D. Maxwell, T. Fernholz, C. S. Adams, I. Lesanovsky, and C. Ates, Phys. Rev. A 88, 043436 (2013).

[14] D. Maxwell, D. J. Szwer, D. Paredes-Barato, H. Busche, J. D. Pritchard, A. Gauguet, K. J. Weatherill, M. P. A. Jones, and C. S. Adams, Phys. Rev. Lett. 110, 103001 (2013).

[15] G. Günter, H. Schempp, M. Robert-de-Saint-Vincent, V. Gavryusev, S. Helmrich, C. S. Hofmann, S. Whitlock, and M. Weidemüller, Science 342, 954 (2013).

[16] A. P. Orioli, A. Signoles, H. Wildhagen, G. Günter, J. Berges, S. Whitlock, and M. Weidemüller, Phys. Rev. Lett. 120, 063601 (2018).

[17] M. Brune, S. Haroche, V. Lefevre, J. M. Raimond, and N. Zagury, Phys. Rev. Lett. 65, 976 (1990).

[18] M. Brune, J. Bernu, C. Guerlin, S. Deléglise, C. Sayrin, S. Gleyzes, S. Kuhr, I. Dotsenko, J. M. Raimond, and S. Haroche, Phys. Rev. Lett. 101, 240402 (2008).

[19] M. Brune, S. Haroche, J. M. Raimond, L. Davidovich, and N. Zagury, Phys. Rev. A 45, 5193 (1992).

[20] S. Gleyzes, S. Kuhr, C. Guerlin, J. Bernu, S. Deléglise, U. B. Hoff, M. Brune, J.-M. Raimond, and S. Haroche, Nature (London) 446, 297 (2007).

[21] S. Deléglise, I. Dotsenko, C. Sayrin, J. Bernu, J.-M. Raimond, and S. Haroche, Nature (London) 455, 510 (2008).

[22] T. Xia, X. L. Zhang, and M. Saffman, Phys. Rev. A 88, 062337 (2013).

[23] M. Saffman, J. Phys. B: At., Mol. Opt. Phys. 49, 202001 (2016).

[24] T. L. Nguyen, J. M. Raimond, C. Sayrin, R. Cortiñas, T. CantatMoltrecht, F. Assemat, I. Dotsenko, S. Gleyzes, S. Haroche, G. Roux, Th. Jolicoeur, and M. Brune, Phys. Rev. X 8, 011032 (2018).
[25] D. A. Anderson, A. Schwarzkopf, R. E. Sapiro, and G. Raithel, Phys. Rev. A 88, 031401(R) (2013).

[26] P. Nussenzveig, F. Bernardot, M. Brune, J. Hare, J. Raimond, S. Haroche, and W. Gawlik, Phys. Rev. A 48, 3991 (1993).

[27] R. J. Brecha, G. Raithel, C. Wagner, and H. Walther, Opt. Commun. 102, 257 (1993).

[28] V. Zhelyazkova and S. Hogan, Phys. Rev. A 94, 023415 (2016).

[29] Z. D. Gaeta and C. R. Stroud, Phys. Rev. A 42, 6308 (1990).

[30] T. P. Hezel, C. E. Burkhardt, M. Ciocca, L. He, and J. J. Leventhal, Am. J. Phys. 60, 329 (1992).

[31] T. P. Hezel, C. E. Burkhardt, M. Ciocca, and J. J. Leventhal, Am. J. Phys. 60, 324 (1992).

[32] I. Samengo, Phys. Rev. A 58, 2767 (1998).

[33] M. Bucher, arXiv:0802.1366.

[34] T. Deeney and C. O'Sullivan, Am. J. Phys. 82, 883 (2014).

[35] I. I. Beterov, I. I. Ryabtsev, D. B. Tretyakov, and V. M. Entin, Phys. Rev. A 79, 052504 (2009).

[36] W. E. Cooke and T. F. Gallagher, Phys. Rev. A 21, 588 (1980).

[37] T. F. Gallagher, Rydberg Atoms (Cambridge University Press, Cambridge, UK, 1994).

[38] F. Robicheaux, J. V. Hernandez, T. Topcu, and L. D. Noordam, Phys. Rev. A 70, 042703 (2004).

[39] N. Šibalić, J. D. Pritchard, C. S. Adams, and K. J. Weatherill, Comp. Phys. Commun. 220, 319 (2017).

[40] S. Patsch, D. M. Reich, J.-M. Raimond, M. Brune, S. Gleyzes, and C. P. Koch, Phys. Rev. A 97, 053418 (2018).

[41] We collect all states that are coupled to the initial state via at most $c=3$ coupling matrix elements. Of these we remove all states that are detuned by more than $E_{\text {cut }}=2000 \mathrm{MHz}$ from the initial state. Finally, we verified that results do not change when these numerical constraints were loosened.

[42] D. Kleppner, Phys. Rev. Lett. 47, 233 (1981).

[43] R. G. Hulet, E. S. Hilfer, and D. Kleppner, Phys. Rev. Lett. 55, 2137 (1985).

[44] J. C. Tully, J. Chem. Phys. 93, 1061 (1990).

[45] J. C. Tully and R. K. Preston, J. Chem. Phys. 55, 562 (1971).

[46] M. Barbatti, Wiley Interdiscip. Rev.: Comput. Mol. Sci. 1, 620 (2011).

[47] K. Leonhardt, Ph.D. thesis, Technische Universität Dresden, 2016, arXiv:1612.07858.

[48] I. S. Gradshteym and I. M. Ryzhik, Table of Integrals, Series and Products (Academic Press, London, UK, 2007).

[49] T. Amthor (2008), thesis online at https://freidok.unifreiburg.de/data/5802.

[50] W. Li, I. Mourachko, M. W. Noel, and T. F. Gallagher, Phys. Rev. A 67, 052502 (2003).

[51] S. Weber, C. Tresp, H. Menke, A. Urvoy, O. Firstenberg, H. P. Büchler, and S. Hofferberth, J. Phys. B 50, 133001 (2017).

[52] K. Singer, J. Stanojevic, M. Weidemüller, and R. Côté, J. Phys. B 38, S295 (2005). 